PROCEEDINGS OF THE

AMERICAN MATHEMATICAL SOCIETY

Volume 131, Number 10, Pages 3181-3189

S 0002-9939(03)06868-0

Article electronically published on February 14, 2003

\title{
HARDY SPACES OF SPACES OF HOMOGENEOUS TYPE
}

\author{
XUAN THINH DUONG AND LIXIN YAN \\ (Communicated by Andreas Seeger)
}

\begin{abstract}
Let $X$ be a space of homogeneous type, and $L$ be the generator of a semigroup with Gaussian kernel bounds on $L^{2}(X)$. We define the Hardy spaces $H_{s}^{p}(X)$ of $X$ for a range of $p$, by means of area integral function associated with the Poisson semigroup of $L$, which is proved to coincide with the usual atomic Hardy spaces $H_{a t}^{p}(X)$ on spaces of homogeneous type.
\end{abstract}

\section{INTRODUCTION}

We begin by recalling the definitions necessary for introducing Hardy spaces on spaces of homogeneous type. A quasi-metric $d$ on a set $X$ is a function

$$
d: X \times X \mapsto[0, \infty)
$$

satisfying:

(i) $d(x, y)=0$ if and only if $x=y$;

(ii) $d(x, y)=d(y, x)$ for all $x, y \in X$;

(iii) there exists a constant $A<\infty$ such that for all $x, y$, and $z \in X, d(x, y) \leq$ $A(d(x, z)+d(z, y))$.

Any quasi-metric defines a topology, for which the balls $B(x, r)=\{y \in X$ : $d(y, x)<r\}$ form a base. However, the balls themselves need not be open when $A>1$.

Definition $1.1(\underline{\mathrm{CW}})$. A space of homogeneous type $(X, d, \mu)$ is a set together with a quasi-metric $d$ and a nonnegative measure $\mu$ on $X$ such that $\mu(B(x, r))<\infty$ for all $x \in X$ and all $r>0$, and there exists $A^{\prime}<\infty$ such that for all $x \in X$ and all $r>0$,

$$
\mu(B(x, 2 r)) \leq A^{\prime} \mu(B(x, r)) .
$$

Here $\mu$ is assumed to be defined on a $\sigma$-algebra which contains all Borel sets and all balls $B(x, r)$.

Received by the editors January 24, 2002 and, in revised form, May 16, 2002.

2000 Mathematics Subject Classification. Primary 42B20, 42B30, 47G10.

Key words and phrases. Spaces of homogeneous type, Hardy spaces, semigroup, Calderón-type reproducing formula, atomic decomposition.

Both authors were partially supported by a grant from Australia Research Council, and the second author was also partially supported by the NSF of China.

(C)2003 American Mathematical Society 
Macias and Segovia [MS have shown that one can replace $d$ by another quasimetric $\rho$ such that there exist $c$ and some $\theta, 0<\theta<1$,

$$
\begin{gathered}
\rho(x, y) \approx \inf \{\mu(B): B \text { is a ball containing } x \text { and } y\} \\
\left|\rho(x, y)-\rho\left(x^{\prime}, y\right)\right| \leq c \rho\left(x, x^{\prime}\right)^{\theta}\left[\rho(x, y)+\rho\left(x^{\prime}, y\right)\right]^{1-\theta} \text { for all } x, x^{\prime} \in X \text { and } y \in X .
\end{gathered}
$$

We will suppose that $\mu(X)=\infty$ and $\mu(\{x\})=0$ for all $x \in X$. A function $a(x)$ defined on $X$ is called a $p$-atom if there exists a ball $B\left(x_{0}, r\right)$ for some $x_{0} \in X$ and $r>0$ such that supp $a(x) \subset B\left(x_{0}, r\right)$,

$$
\|a\|_{2} \leq \mu\left(B\left(x_{0}, r\right)\right)^{1 / 2-1 / p} \text { and } \quad \int_{X} a(x) d \mu(x)=0 .
$$

Definition $1.2([\mathrm{CW}])$. We say that a function $f(x) \in H_{a t}^{p}(X)$ for $(1+\theta)^{-1}<p \leq$ 1 , if $f(x)$ possesses an atomic decomposition $f(x)=\sum_{k} \lambda_{k} a_{k}(x)$, where the $a_{k}$ 's are $p$-atoms and $\sum_{k}\left|\lambda_{k}\right|^{p}<\infty$. The norm of $f$ is then defined by

$$
\|f\|_{H_{a t}^{p}(X)}=\inf \left\{\left(\sum_{k}\left|\lambda_{k}\right|^{p}\right)^{1 / p}: \text { for all } f(x)=\sum_{k} \lambda_{k} a_{k}(x)\right\} .
$$

It is well-known that Hardy spaces $H^{p}\left(\mathbb{R}^{n}\right)$ on $\mathbb{R}^{n}$ can be characterized by means of the square function associated with the Poisson semigroup $e^{-t \sqrt{A}}$ or the heat semigroup $e^{-t A}$, where $A=-\triangle$ is the Laplace operator; see [FeS]. In this paper, our concern is to establish similar results on spaces of homogeneous type under the following assumptions about the operator $L$ :

(1.1) $L$ is a self-adjoint, positive definite operator on $L^{2}(X)$;

(1.2) the kernel of $e^{-t L}$, denoted by $K_{t}(x, y)$, is a measurable function on $X \times X$ and there exists $\beta>0$ such that for all $0<t<\infty$ and almost every $x, y \in X$,

$$
\left|K_{t}(x, y)\right| \leq \frac{C}{\mu(B(x, \sqrt{t}))} \exp \left\{-\frac{\beta d^{2}(x, y)}{t}\right\} ;
$$

(1.3) for all $y \in X$ and all $0<t<\infty$, the function $x \rightarrow K_{t}(x, y)$ is Hölder continuous in $X$ and there exists $\gamma>0$ such that for all $0<t<\infty$ and all $x, x^{\prime}, y \in X$,

$$
\left|K_{t}(x, y)-K_{t}\left(x^{\prime}, y\right)\right| \leq \frac{C}{\mu(B(x, \sqrt{t}))}\left(\frac{d\left(x, x^{\prime}\right)}{\sqrt{t}}\right)^{\gamma} ;
$$

(1.4) for all $t>0, e^{-t L}(1)=1$.

We define the square function $S_{\alpha}(f), 0<\alpha<\infty$ by the area integral

$S_{\alpha}(f)(x)=\left(\int_{0}^{\infty} \int_{\rho(x, y)<\alpha t} \mu(B(x, \alpha t))^{-1}\left|t \frac{\partial}{\partial t} e^{-t \sqrt{L}}(f)\right|^{2} \frac{d \mu(y) d t}{t}\right)^{1 / 2}, \quad x \in X$.

$S_{1}(f)(x)$ will be denoted by $S(f)(x)$. By a standard argument, for any $0<\alpha<\infty$ we have

$$
\left\|S_{\alpha}(f)\right\|_{L^{p}(X)} \approx\left\|S_{1}(f)\right\|_{L^{p}(X)}, \quad 0<p<\infty .
$$

Definition 1.3. We say that a function $f(x) \in H_{s}^{p}(X),(1+\gamma)^{-1}<p \leq 1$, if $S(f)(x)$ belongs to $\in L^{p}(X)$, and its norm is defined by $\|f\|_{H_{s}^{p}(X)}=\|S(f)\|_{L^{p}(X)}$. 
The main result of this paper is the following theorem.

Theorem 1.4. Assume that $L$ satisfies the conditions (1.1), (1.2), (1.3) and (1.4). Then, for any $p$ such that $\max \left((1+\theta)^{-1},(1+\gamma)^{-1}\right)<p \leq 1, H_{s}^{p}(X)=H_{a t}^{p}(X)$ with equivalent norms.

The paper is organized as follows. In Section 2, we introduce a Calderón-type reproducing formula associated with the self-adjoint operator $L$. Our reproducing formula (2.5) below is based on the functional calculus of $L$. For other constructions of Calderón-type reproducing formula on spaces of homogeneous type, we refer readers to $[\mathrm{HS}$. Using our reproducing formula we obtain atomic decomposition of Hardy spaces $H_{s}^{p}(X)$, and prove Theorem 1.4 in Section 3. We give some applications in Section 4.

\section{CALDERÓN-TYPe REPRODUCING FORMUlA}

Recall that for any positive self-adjoint operator $L$ on $L^{2}(X)$ and for every bounded Borel function $F:[0, \infty) \mapsto \mathcal{C}$, we define an operator $F(L): L^{2}(X) \mapsto$ $L^{2}(X)$ by the formula

$$
F(L)=\int_{0}^{\infty} F(\lambda) d E(\lambda)
$$

where $E(\lambda)$ is the spectral decomposition of the operator $L$. Therefore, the operators $\cos (t \sqrt{L})$ and $(t \sqrt{L})^{-1} \sin (t \sqrt{L})$ are well-defined on $L^{2}(X)$. The functional calculus for $L$ gives us the relationship

$$
(t \sqrt{L})^{-1} \sin (t \sqrt{L})=(2 t)^{-1} \int_{-t}^{t} \cos (\xi \sqrt{L}) d \xi .
$$

See Theorem 2 in Si]. Throughout the paper, we denote

$$
S_{t}(\sqrt{L})=(t \sqrt{L})^{-1}(2 \sin (t \sqrt{L} / 2)-\sin (t \sqrt{L})) .
$$

Lemma 2.1. Assume that L satisfies (1.1) and (1.2). Then the Schwartz kernel $K_{S_{t}(\sqrt{L})}$ of $S_{t}(\sqrt{L})$ has support contained in

$$
\left\{(x, y) \in X^{2}: d(x, y) \leq t\right\} .
$$

Proof. An argument of Davies, as adapted in [DR, shows that the semigroup $e^{-t L}$ can be extended to an analytic semigroup for all $z$ such that $\operatorname{Re} z \geq 0$, and

$$
\left|K_{z}(x, y)\right| \leq \frac{C}{\mu(B(x, \sqrt{\operatorname{Re} z}))} \exp \left\{-\frac{\beta d^{2}(x, y)}{\operatorname{Re} z}\right\} .
$$

See Lemma 2.4 in [CD]. Then it follows from Theorem 3 in Si] that we have

$$
\operatorname{supp}\left(K_{\cos (t \sqrt{L})}\right) \subseteq\left\{(x, y) \in X^{2}: d(x, y) \leq t\right\} .
$$

Using the formula (2.1), we get

$$
\operatorname{supp}\left(K_{(t \sqrt{L})^{-1} \sin (t \sqrt{L})}\right) \subseteq\left\{(x, y) \in X^{2}: d(x, y) \leq t\right\}
$$

which implies this lemma.

Lemma 2.2. Assume that $L$ satisfies (1.1), (1.2) and (1.4). For all $t>0$, we have $S_{t}(\sqrt{L})(1)=0$. 
Proof. By (2.2) it suffices to prove $(t \sqrt{L})^{-1} \sin (t \sqrt{L})(1)=1$ for all $t>0$. For each $\epsilon>0$, denote $\theta=2 \arctan \left(\frac{\epsilon}{2}\right)$. Let $\Gamma_{b}^{\epsilon}=\Gamma_{0, b}^{\epsilon} \cup \Gamma_{1, b}^{\epsilon}$ be the oriented contour, which is given by $\Gamma_{0, b}^{\epsilon}=\left\{\lambda=b e^{i \phi}, \phi \in[-\pi, \pi) \backslash(-\theta, \theta)\right\}$ for some $b>0$, and

$$
\Gamma_{1, b}^{\epsilon}:=\left\{\begin{array}{l}
-t e^{-i \theta}, \quad-\infty<t \leq-b, \\
t e^{i \theta}, \quad b \leq t<\infty .
\end{array}\right.
$$

We can write (see e.g. $\underline{\mathrm{MC}}$ )

$$
e^{i t \sqrt{L}} e^{-\epsilon t \sqrt{L}}=(2 \pi i)^{-1} \int_{\Gamma_{b}^{\epsilon}}(\lambda I-L)^{-1} e^{i t \sqrt{\lambda}} \cdot e^{-\epsilon t \sqrt{\lambda}} d \lambda .
$$

The integral is absolutely convergent in the norm topology on $L^{2}(X)$. It is also independent of the choice of $b$.

The assumption (1.4) implies that $(\lambda I-L)^{-1}(1)=\lambda^{-1}$. As a consequence, we have

$$
\begin{aligned}
& \lim _{\epsilon \rightarrow 0} e^{i t \sqrt{L}} e^{-\epsilon t \sqrt{L}}(1)=\lim _{\epsilon \rightarrow 0} \lim _{b \rightarrow 0}(2 \pi i)^{-1} \int_{\Gamma_{b}^{\epsilon}} \lambda^{-1} e^{i t \sqrt{\lambda}} \cdot e^{-\epsilon t \sqrt{\lambda}} d \lambda \\
& =\lim _{\epsilon \rightarrow 0} \lim _{b \rightarrow 0}(2 \pi i)^{-1}\left(\int_{\Gamma_{0, b}^{\epsilon}} \lambda^{-1} e^{i t \sqrt{\lambda}} \cdot e^{-\epsilon t \sqrt{\lambda}} d \lambda+\int_{\Gamma_{1, b}^{\epsilon}} \lambda^{-1} e^{i t \sqrt{\lambda}} \cdot e^{-\epsilon t \sqrt{\lambda}} d \lambda\right) \\
& =1+\lim _{\epsilon \rightarrow 0} \lim _{b \rightarrow 0}(2 \pi i)^{-1} \int_{\Gamma_{1, b}^{\epsilon}} \lambda^{-1} e^{i t \sqrt{\lambda}} \cdot e^{-\epsilon t \sqrt{\lambda}} d \lambda .
\end{aligned}
$$

Since $\theta=2 \arctan \left(\frac{\epsilon}{2}\right)$, it can be verified that

$$
\begin{aligned}
& \lim _{\epsilon \rightarrow 0} \lim _{b \rightarrow 0} \operatorname{Re}\left[(2 \pi i)^{-1} \int_{\Gamma_{1, b}^{\epsilon}} \lambda^{-1} e^{i t \sqrt{\lambda}} \cdot e^{-\epsilon t \sqrt{\lambda}} d \lambda\right] \\
& \quad=\lim _{\epsilon \rightarrow 0} \pi^{-1} \int_{0}^{\infty} e^{-x} \cdot\left[\sin \left(\frac{2}{\epsilon}+\epsilon\right) x-\sin \left(\frac{2}{3 \epsilon}-\frac{\epsilon}{3}\right) x\right] \frac{d x}{x} \\
& \quad=-1 / 2+1 / 2=0 .
\end{aligned}
$$

Substituting (2.4) into (2.3) yields

$$
\cos (t \sqrt{L})(1)=\operatorname{Re}\left[e^{i t \sqrt{L}}(1)\right]=\lim _{\epsilon \rightarrow 0} \operatorname{Re}\left[e^{i t \sqrt{L}} e^{-\epsilon t \sqrt{L}}(1)\right]=1
$$

for all $t>0$. Hence,

$$
\begin{aligned}
(t \sqrt{L})^{-1} \sin (t \sqrt{L})(1) & =(2 t)^{-1} \int_{-t}^{t} \cos (\xi \sqrt{L})(1) d \xi \\
& =(2 t)^{-1} \int_{-t}^{t} d \xi=1,
\end{aligned}
$$

which implies $S_{t}(\sqrt{L})(1)=0$ for all $t>0$.

Lemma 2.3. Assume that $L$ satisfies (1.1). Then for any $f \in L^{2}(X)$, there exists a constant $C$ such that

$$
\left(\int_{0}^{\infty}\left\|S_{t}(\sqrt{L})(f)\right\|_{2}^{2} \frac{d t}{t}\right)^{1 / 2} \leq C\|f\|_{L^{2}(X)} .
$$


Proof. Let $\psi(t)=2(\sqrt{t})^{-1} \sin (\sqrt{t} / 2)-(\sqrt{t})^{-1} \sin (\sqrt{t})$. Then

$$
q=(\sqrt{2})^{-1}\left(\int_{0}^{\infty}|\psi(t)|^{2} \frac{d t}{t}\right)^{1 / 2}<\infty .
$$

Since $L$ is a self-adjoint operator, we have

$$
\begin{aligned}
\left(\int_{0}^{\infty}\left\|S_{t}(\sqrt{L})(f)\right\|_{2}^{2} \frac{d t}{t}\right)^{1 / 2} & =\left(\int_{0}^{\infty}\left\|\psi\left(t^{2} L\right)(f)\right\|_{2}^{2} \frac{d t}{t}\right)^{1 / 2} \\
& \leq q\|f\|_{L^{2}(X)} .
\end{aligned}
$$

Next, we establish a new Calderón-type reproducing formula associated with the semigroup generated by $L$, which will be used in the next section.

Lemma 2.4. Assume that $L$ satisfies (1.1). Then, for any $f(x) \in L^{2}(X)$, we have

$$
f(x)=C_{s} \int_{0}^{\infty} S_{t}(\sqrt{L})\left(t \frac{\partial}{\partial t}\right) e^{-t \sqrt{L}}(f) \frac{d t}{t}(x),
$$

where the integral converges strongly in $L^{2}(X)$, and $C_{s}=\left(\arctan \left(\frac{4}{3}\right)-\frac{\pi}{4}\right)^{-1}$.

Proof. Note that for any $\lambda>0$, we have

$$
\int_{0}^{\infty} S_{t}(\sqrt{\lambda})(t \sqrt{\lambda}) e^{-t \sqrt{\lambda}} \frac{d t}{t}=\int_{0}^{\infty}\left(2 e^{-2 t}-e^{-t}\right) \sin t \frac{d t}{t}=C_{s}^{-1} .
$$

As a consequence, one has

$$
f(x)=C_{s} \int_{0}^{\infty} S_{t}(\sqrt{L})\left(t \frac{\partial}{\partial t}\right) e^{-t \sqrt{L}}(f) \frac{d t}{t}(x),
$$

where $C_{s}=\left(\arctan \left(\frac{4}{3}\right)-\frac{\pi}{4}\right)^{-1}$, and the integral converges strongly in $L^{2}(X)$.

\section{Proof of Theorem 1.4}

To prove Theorem 1.4, we first recall a result of Christ Ch which gives an analogue of the Euclidean dyadic cubes.

Lemma 3.1. There exist a collection of open subsets $\left\{Q_{\tau}^{k} \subset X: k \in Z, \tau \in I_{k}\right\}$, where $I_{k}$ denotes some (possibly finite) index set depending on $k$, and constants $\delta \in(0,1), a_{0}>0, \eta>0$ and $0<c_{1}, c_{2}<\infty$ such that

(i) $\mu\left(X \backslash \cup Q_{\tau}^{k}\right)=0$ for all $k \in Z$;

(ii) if $j \geq k$, then either $Q_{\tau^{\prime}}^{j} \subset Q_{\tau}^{k}$ or $Q_{\tau^{\prime}}^{j} \cap Q_{\tau}^{k}=\emptyset$;

(iii) for all $(k, \tau)$ and $j<k$, there is a unique $\tau^{\prime}$ such that $Q_{\tau}^{k} \subset Q_{\tau}^{j}$;

(iv) $\operatorname{diam}\left(Q_{\tau}^{k}\right) \leq c_{1} \delta^{k}$;

(v) each $Q_{\tau}^{k}$ contains some ball $B\left(z_{\tau}^{k}, a_{0} \delta^{k}\right)$.

We fix such a collection of open subsets as in Lemma 3.1 and call all $Q_{\tau}^{k}$ in Lemma 3.1 the dyadic cubes in $X$. Without loss of generality, we may assume that $\delta=1 / 2$ in Lemma 3.1. It is easy to check that our results and proofs are independent of the choice of open subsets which satisfy the hypotheses of Lemma 3.1 .

We first prove that $H_{s}^{p}(X) \subset H_{a t}^{p}(X)$. We follow the idea in [CF]. Let $\Omega_{k}=$ $\left\{x \in X: S_{c_{0}}(f)(x)>2^{k}\right\}$, where $c_{0}$ is a constant to be chosen later. Set

$$
\mathcal{B}_{k}=\left\{Q: \mu\left(Q \cap \Omega_{k}\right)>\mu(Q) / 2 \text { and } \mu\left(Q \cap \Omega_{k+1}\right)<\mu(Q) / 2\right\} .
$$


Note that for each dyadic cube $Q$ in $X$ there is a unique $k \in \mathbf{Z}$ such that $Q \in \mathcal{B}_{k}$. For each dyadic cube $Q \in \mathcal{B}_{k}$, there is a unique maximal dyadic cube $Q^{\prime} \in \mathcal{B}_{k}$ such that $Q \subseteq Q^{\prime}$. Denote the collection of all maximal dyadic cubes in $\mathcal{B}_{k}$ by $Q_{k}^{i}, i \in I_{k}$, an index set which depends on $k$ (it is possibly finite). We then have

$$
\bigcup_{Q: \text { dyadic cube }} Q=\bigcup_{k} \bigcup_{i \in I_{k}} \bigcup_{Q \subseteq Q_{k}^{i}, Q \in \mathcal{B}_{k}} Q
$$

For dyadic cube $Q$ with $\mu(Q) \sim 2^{-k}$, we write $\hat{Q}=\left\{(y, t) \in X \times \mathbb{R}^{+}, y \in Q, 2^{k}<\right.$ $\left.t<2^{k+1}\right\}$, and for any $k$ and $i$, let $\hat{Q}_{k}^{i}=\bigcup_{\substack{Q \subset Q_{k}^{i} \\ Q \in \mathcal{B}_{k}}} \hat{Q}$. If $f \in H_{s}^{p}(X) \cap L^{2}(X)$, applying the Calderón-type reproducing formula $(2.5)$ we have

$$
\begin{aligned}
f(x) & =\lim _{A \rightarrow \infty} \lim _{\epsilon \rightarrow 0} C_{s} \int_{\epsilon}^{A} S_{t}(\sqrt{L})\left(t \frac{\partial}{\partial t}\right) e^{-t \sqrt{L}}(f) \frac{d t}{t}(x) \\
& =\sum_{k} \sum_{\mu(Q) \sim 2^{-k}} C_{s} \int_{2^{k}}^{2^{k+1}} \int_{Q} S_{t}(x, y)\left(t \frac{\partial}{\partial t}\right) e^{-t \sqrt{L}}(f)(y) \frac{d \mu(y) d t}{t} \\
& =\sum_{k, i} \sum_{Q \subseteq Q_{k}^{i}, Q \in \mathcal{B}_{k}} C_{s} \int_{\hat{Q}} S_{t}(x, y)\left(t \frac{\partial}{\partial t}\right) e^{-t \sqrt{L}}(f)(y) \frac{d \mu(y) d t}{t} \\
& =\sum_{k, i} C_{s} \int_{\hat{Q}_{k}^{i}} S_{t}(x, y)\left(t \frac{\partial}{\partial t}\right) e^{-t \sqrt{L}}(f)(y) \frac{d \mu(y) d t}{t} .
\end{aligned}
$$

Set

$$
\lambda_{k, i}=c \mu\left(Q_{k}^{i}\right)^{1 / p-1 / 2}\left(\int_{\hat{Q}_{k}^{i}}\left|\left(t \frac{\partial}{\partial t}\right) e^{-t \sqrt{L}}(f)\right|^{2} \frac{d y d t}{t}\right)^{1 / 2} .
$$

Then one can write (3.1) as

$$
f(x)=\sum_{k, i} \lambda_{k, i} a_{k, i}(x)
$$

where

$$
a_{k, i}(x)=C_{s} \lambda_{k, i}^{-1} \int_{\hat{Q}_{k}^{i}} S_{t}(x, y)\left(t \frac{\partial}{\partial t}\right) e^{-t \sqrt{L}}(f)(y) \frac{d \mu(y) d t}{t} .
$$

We now show that this sum (3.2) gives a decomposition of $f$ into atoms. Since

$$
\operatorname{supp}\left(K_{S_{t}(\sqrt{L})}\right) \subset\left\{(x, y) \in X^{2}: d(x, y)<t\right\}
$$

by Lemma 2.1, we have

$$
\operatorname{supp} a_{k, i}(x) \subset \bigcup_{\substack{Q \subset Q_{k}^{i} \\ Q \in \mathcal{B}_{k}}} Q \subset 2 Q_{k}^{i} .
$$


Using Lemma 2.3, we have

$$
\begin{aligned}
& \left\|a_{k, i}\right\|_{2} \leq \sup _{\|b\|_{2} \leq 1}\left|\int_{X} a_{k, i}(x) b(x) d \mu(x)\right| \\
\leq & C\left(\lambda_{k, i}\right)^{-1} \sup _{\|b\|_{2} \leq 1}\left(\int_{0}^{\infty} \int_{X}\left(t \frac{\partial}{\partial t}\right) e^{-t \sqrt{L}}(f)(y) \chi_{\hat{Q}_{k, i}}(y, t)\left(S_{t}(\sqrt{L})^{*}\right)(b)(y) \frac{d \mu(y) d t}{t}\right) \\
\leq & C \mu\left(Q_{k}^{i}\right)^{1 / 2-1 / p} \sup _{\|b\|_{2} \leq 1}\left(\int_{0}^{\infty} \int_{X}\left|S_{t}(\sqrt{L})(b)(y)\right|^{2} \frac{d \mu(y) d t}{t}\right)^{1 / 2} \\
\leq & C \mu\left(Q_{k}^{i}\right)^{1 / 2-1 / p} .
\end{aligned}
$$

It follows from Lemma 2.2 that

$$
\int_{X} a_{k, i}(x) d \mu(x)=\lambda_{k, i}^{-1} \int_{\hat{Q}_{k}^{i}} S_{t}^{*}(\sqrt{L})(1)\left(t \frac{\partial}{\partial t}\right) e^{-t \sqrt{L}}(f)(y) \frac{d \mu(y) d t}{t}=0 .
$$

This proves that for each $k, i$, the function $a_{k, i}(x)$ is an atom.

Now we estimate $\sum_{k, i}\left|\lambda_{k, i}\right|^{p}$. We need the following lemma, whose proof is similar to that of Lemma 7.18 in [CF].

Lemma 3.2. Suppose that $\mathcal{B}_{k}$ is as above. Then there exists a constant $C$ such that

$$
\begin{aligned}
\sum_{Q \in \mathcal{B}_{k}} \int_{\hat{Q}}\left|\left(t \frac{\partial}{\partial t}\right) e^{-t \sqrt{L}}(f)(y)\right|^{2} \frac{d \mu(y) d t}{t} & =\sum_{i} \int_{\hat{Q}_{k}^{i}}\left|\left(t \frac{\partial}{\partial t}\right) e^{-t \sqrt{L}}(f)(y)\right|^{2} \frac{d \mu(y) d t}{t} \\
& \leq C 2^{2 k} \mu\left(\Omega_{k}\right)
\end{aligned}
$$

Using Lemma 3.2 and the Hölder inequality we have

$$
\begin{aligned}
\sum_{k, i}\left|\lambda_{k, i}\right|^{p} & \leq \sum_{k} \sum_{i} \mu\left(Q_{k}^{i}\right)^{1-p / 2}\left(\int_{\hat{Q}_{k}^{i}}\left|\left(t \frac{\partial}{\partial t}\right) e^{-t \sqrt{L}}(f)\right|^{2} \frac{d \mu(y) d t}{t}\right)^{p / 2} \\
& \leq \sum_{k}\left(\sum_{i} \mu\left(Q_{k}^{i}\right)\right)^{1-p / 2}\left(\sum_{i} \int_{\hat{Q}_{k}^{i}}\left|\left(t \frac{\partial}{\partial t}\right) e^{-t \sqrt{L}}(f)\right|^{2} \frac{d \mu(y) d t}{t}\right)^{p / 2} \\
& \leq C \sum_{k} \mu\left(\Omega_{k}\right)^{1-p / 2}\left(2^{2 k} \mu\left(\Omega_{k}\right)\right)^{p / 2} \leq C \sum_{k} 2^{k p} \mu\left(\Omega_{k}\right) \\
& \leq C\left\|S_{c_{0}}(f)\right\|_{L^{p}(X)}^{p} \leq C\|S(f)\|_{L^{p}(X)}^{p} .
\end{aligned}
$$

This proves that a function $f \in H_{s}^{p}(X) \cap L^{2}$ possesses an atomic decomposition. A standard density argument gives an atomic decomposition for $f \in H_{s}^{p}(X)$. See, for example, $\mathrm{CF}$.

We now prove that $H_{a t}^{p}(X) \subset H_{s}^{p}(X)$. Let $a(x)$ be an atom supported in a cube $Q$. Note that $L$ satisfies assumptions (1.2) and (1.3), a standard argument shows that $\|a\|_{H_{s}^{p}} \leq C$, hence $H_{a t}^{p}(X) \subset H_{s}^{p}(X)$. So, the proof of Theorem 1.4 is completed.

\section{Applications}

Example 4.1. Let $\Phi: \mathbb{R}^{n-1} \longmapsto \mathbb{R}^{n}$ be a Lipschitz function, i.e., $\|\nabla \Phi\|_{\infty} \leq M<$ $\infty$ for some constant $M$. Define $\Omega=\left\{(X, y) \mid \in \mathbb{R}^{n-1} \times \mathbb{R}^{n} ; y>\Phi(X)\right\}$; then $\Omega$ is a Lipschitz domain of $\mathbb{R}^{n}$. 
We assume that $a_{i j}(x)=a_{j i}(x)$ are bounded, real-valued, Lebesgue measurable functions on $\Omega$, which satisfy the ellipticity condition

$$
\lambda|\xi|^{2} \leq \sum_{i, j=1}^{n} a_{i j}(x) \xi_{1} \xi_{j} \leq \lambda^{-1}|\xi|^{2}
$$

for some constant $\lambda \in(0,1)$, and for all $x \in \Omega$ and $\xi \in \mathbb{R}^{n}$. A function $u$ belonging to the Sobolev space $W^{1,2}(\Omega)$ is called a solution of the Neumann boundary value problem

$$
\begin{array}{ll}
L u=f & \text { in } \Omega, \\
\sum_{i, j=1}^{n} a_{i j} \nu_{j} \frac{\partial u}{\partial x_{i}}=0 & \text { on } \partial \Omega,
\end{array}
$$

where $\nu_{j}, 1 \leq j \leq n$, are the components of the unit outer normal vector field on $\partial \Omega$, if $u$ satisfies

$$
\int_{\Omega} \sum_{i, j=1}^{n} a_{i j} \frac{\partial u}{\partial x_{j}} \frac{\partial v}{\partial x_{i}} d x=\int_{\Omega} f v d x
$$

for all nonnegative functions $v \in C^{1}(\Omega)$. See, for example, $\mathrm{D}$.

Then the operator $L$ satisfies all assumptions in Theorem 1.4. Recall that Hardy spaces $H_{z}^{p}(\Omega)$ of D-C. Chang, S.G. Krantz and E.M. Stein ( $\mathrm{CKS}$ ) on the Lipschitz domain of $\mathbb{R}^{n}$ is given by

$$
H_{z}^{p}(\Omega)=H^{p}\left(\mathbb{R}^{n}\right) \cap\left\{f \in H^{p}\left(\mathbb{R}^{n}\right): f=0 \text { on }{ }^{c}(\bar{\Omega})\right\} /\left\{f \in H^{p}\left(\mathbb{R}^{n}\right): f=0 \text { on } \Omega\right\} .
$$

By Theorem 3.3 in CKS], Theorem 1.4 gives a new proof of $H_{s}^{p}(\Omega)=H_{z}^{p}(\Omega)$. See also $\mathrm{AR}$.

Example 4.2. Let $\mathbf{g}$ be a finite dimensional nilpotent Lie algebra. Assume that

$$
\mathbf{g}=\bigoplus_{i=1}^{m} \mathbf{g}_{i}
$$

as a vector space, where $\left[\mathbf{g}_{i}, \mathbf{g}_{i}\right] \subseteq \mathbf{g}_{i+j}$ for all $i, j$, and $\mathbf{g}_{1}$ generates $\mathbf{g}$ as a Lie algebra.

Let $G$ be the associated connected, simply connected Lie group. Then $G$ has homogeneous dimension $d$ given by the formula

$$
d=\sum_{j=1}^{m} j \operatorname{dim}\left(\mathbf{g}_{j}\right),
$$

where $\operatorname{dim}\left(\mathbf{g}_{j}\right)$ denotes the dimension of $\mathbf{g}_{j}$.

Consider any finite subset $\left\{X_{k}\right\}$ of $\mathbf{g}_{1}$ which spans $\mathbf{g}_{1}$. Each $X_{k}$ can be identified with a unique left invariant vector field on $G$. Let $d$ be the control distance corresponding to the vector fields and $\rho$ the Haar measure. Then the space $(G, d, \rho)$ is of polynomial growth, hence it is a space of homogeneous type. Define

$$
L=-\sum_{k} X_{k}^{2} \text {. }
$$

The operator $L$ is a left invariant second order differential operator, which is a non-negative self-adjoint operator in $L^{2}(G)$. Moreover, the operator $L$ then has a heat kernel of $e^{-t L}$ which satisfies all assumptions in Theorem 1.4. See [DR, 
and $[\mathrm{Sc}]$. Hence, Theorem 1.4 gives an equivalent characterization of Hardy spaces $H^{p}(G)$ on $G$ (see [FoS] ) by means of the square function associated with the Poisson semigroup.

\section{ACKNOWLEDGMENT}

We thank A. McIntosh and A. Sikora for their useful suggestions.

\section{REFERENCES}

[AR] P. Auscher and E. Russ, Hardy spaces and divergence operators on strongly Lipschitz domain of $\mathbb{R}^{n}$, preprint.

[CD] T. Coulhon and X.T. Duong, Maximal regularity and kernel bounds: observations on a theorem by Hieber and Prüss, Adv. Differential Equations, 5 (2000), 343-368. MR 2001d:34087

[CF] S.Y.A. Chang and R. Fefferman, The Calderón-Zygmund decomposition on product domain, Amer. J. Math., 104 (1982), 445-468. MR 84a:42028

[Ch] M. Christ, A Tb theorem with remarks on analytic capacity and the Cauchy integral, Colloq. Math., 61 (1990), 601-628. MR 92k:42020

[CKS] D-C. Chang, S.G. Krantz, and E.M. Stein, $H^{p}$ theory on a smooth domain in $\mathbb{R}^{n}$ and elliptic boundary value problems, J. Funct. Anal., 114 (1993), 286-347. MR 94j:46032

[CW] R. Coifman and G. Weiss, Extensions of Hardy spaces and their use in analysis, Bull. Amer. Math. Soc., 83 (1977), 569-645. MR 56:6264

[D] E.B. Davies, Heat Kernels and Spectral Theorey, Cambridge Univ. Press, 1989. MR 90e:35123

[DR] X.T. Duong and D.W. Robinson, Semigroup kernels, Poisson bounds, and holomorphic functional calculus, J. Funct. Anal., 142 (1996), 89-128. MR 97j:47056

[FeS] C. Fefferman and E.M. Stein, $H^{p}$ spaces of several variables, Acta Math., 129 (1972), 137-195. MR 56:6263

[FoS] G.B. Folland and E.M. Stein, Hardy spaces on homogeneous group, Math. Notes, 28, Princeton University Press, (1982). MR 84h:43027

[HS] Y.S. Han, E. Sawyer, Littlewood-Paley theory on spaces of homogeneous type and the classical function spaces, Mem. Amer. Math. Soc. 110 (1994), no. 530. MR 96a:42016

[Mc] A. McIntosh, Operators which have an $H_{\infty}$-calculus, Miniconference on Operator Theory and Partial Differential Equations (Proc. Centre Math. Analysis, 14, A.N.U., Canberra, 1986), 210-231. MR 88k:47019

[MS] R.A Maciac and C. Segovia, Lipschitz functions on spaces of homogeneous type, Adv. in Math., 33 (1979), 257-270.

[Sc] L. Saloff-Coste, Analyse sur les groupes de Lie à croissance polynômiale, Arkiv för Mat., 28 (1990), 315-331. MR 92d:22014

[Si] A. Sikora, Sharp pointwise estimates on heat kernels, Quart. J. Math. Oxford, 47 (1996), 371-382. MR 97m:58189

Department of Mathematics, Macquarie University, New South Wales 2109, AusTRALIA

E-mail address: duong@ics.mq.edu.au

Department of Mathematics, Macquarie University, New South Wales 2109, Australia - and - Department of Mathematics, Zhongshan University, Guangzhou, 10275, People's Republic of China

E-mail address: lixin@ics.mq.edu.au 